\title{
ID3 may protect mice from anti-GBM glomerulonephritis by regulating the differentiation of Th17 and Treg cells
}

\author{
HUAN ZHOU ${ }^{1,2}$, LE WANG $^{1}$, QING XU ${ }^{1}$, QINGQUAN LIU ${ }^{1}$, HUI LIU ${ }^{1}$, \\ WENHUI QIU ${ }^{1}$, TINGYANG HU ${ }^{1}$, YONGMAN LV ${ }^{1 *}$ and QIAN ZHANG ${ }^{1 *}$ \\ ${ }^{1}$ Division of Nephrology, Department of Internal Medicine, Tongji Hospital, Tongji Medical College, \\ Huazhong University of Science and Technology, Wuhan, Hubei 430030; ${ }^{2}$ Division of Nephrology, \\ Department of Internal Medicine, Fifth Hospital of Wuhan, Wuhan, Hubei 430050, P.R. China
}

Received January 9, 2017; Accepted July 31, 2017

DOI: $10.3892 / \mathrm{mmr} .2017 .7724$

\begin{abstract}
Anti-glomerular basement membrane glomerulonephritis (anti-GBM GN) is an autoimmune disease that leads to severe and rapidly progressive renal injury. Inhibition of DNA-binding factor 3 (ID3) serves a key role in autoimmune diseases, such as asthma and Sjögren's syndrome, and in experimental allergic encephalitis models. However, the role of ID3 in the progression of anti-GBM GN remains unknown. In the present study, ID3 mRNA expression increased between 3- and 20 -fold in the renal tissues of anti-GBM GN mice compared with the Control group, with a peak at day 14 post-induction. In addition, ID3 protein expression was upregulated from day 7 onwards. The expression of ID3 was also examined in the spleen, and was demonstrated to be increased in the spleen of nephritic mice. $\mathrm{T}$ helper 17 (Th17) cells and regulatory $\mathrm{T}$ (Treg) cells were present throughout the entire period of observation (from day 7 to day 28) in anti-GBM GN mice, which may vary at different time points, accompanied with the expression of ID3. In vitro, ID3 expression was increased when $\mathrm{CD} 4{ }^{+} \mathrm{T}$ cells differentiated into Tregs; however, expression was lower
\end{abstract}

Correspondence to: Dr Qian Zhang, Division of Nephrology, Department of Internal Medicine, Tongji Hospital, Tongji Medical College, Huazhong University of Science and Technology, 1095 Jiefang Avenue, Wuhan, Hubei 430030, P.R. China

E-mail: bearaly@126.com

${ }^{*}$ Contributed equally

Abbreviations: anti-GBM GN, anti-glomerular basement membrane glomerulonephritis; bHLH, basic helix-loop-helix; GBM, glomerular basement membrane; ID3, inhibitor of DNA binding 3; IP, immunoprecipitation; MACS, magnetic-activated cell sorting; PAS, periodic acid-Schiff; RPGN, rapidly progressive glomerulonephritis; SCR, serum creatinine; Tregs, regulatory T cells

Key words: inhibitor of DNA binding 3, E2A, T helper 17 cells, regulatory $\mathrm{T}$ cells, anti-glomerular basement membrane glomerulonephritis in Th17 cells. Following treatment with ID3 small interfering RNA, RAR-related orphan receptor $\gamma \mathrm{t}$, but not forkhead box P3, expression increased. Furthermore, increased expression of interleukin-17A was also observed when ID3 was blocked. In addition, ID3 was able to interact with transcription factor E2A. A significant increase in binding between ID3 and E2A was observed in anti-GBM GN from day 7 onwards, with a peak at day 14 in both renal tissue and spleen. In conclusion, ID3 may be involved in the differentiation of Th17 and Tregs by downregulating Th17 cells, which is probably associated with binding to E2A. The present results suggested that ID3 may offer protection against anti-GBM GN in mice.

\section{Introduction}

Anti-glomerular basement membrane glomerulonephritis (anti-GBM GN) is an aggressive form of autoimmune diseases of the rapidly progressive glomerulonephritis (RPGN) category, which is characterized by a rapid decline in renal function and the formation of glomerular crescents (1). Previous studies have reported that $\mathrm{CD} 4^{+} \mathrm{T}$ cell-mediated immunity serves an important role in the pathogenesis of anti-GBM GN (2-5). CD4 ${ }^{+} \mathrm{T}$ cells may differentiate into four major subsets, including T helper (Th)1, Th2, Th17 and regulatory $\mathrm{T}$ (Tregs) cells, as defined by their pattern of cytokine production and function (6-8). Our previous study revealed that anti-GBM GN was driven by Th1 cell-mediated immune response and Th17 cells also contributed to the progress of anti-GBM GN (4). However, the combined actions of Th1 and Th17 cells lead to renal injury from a number of activated mechanisms. The immunopathology of Th17 cell-mediated immune response develops early and may be followed later by Th1 cell-mediated injury (9). Tregs are a special subset of T cell that serve a protective role in anti-GBM GN and significantly reduce renal injury (10). The activity of Th1 and Th17 cells may be attenuated by the anti-inflammatory actions of Tregs, which inhibits $\mathrm{T}$ cell proliferation and $\mathrm{T}$ effector function (11). Although Th17 cells and Tregs serve different functions, they have the same precursor cells, which may be differentiated to different cell types through pleiotropic cytokines $(12,13)$. Interleukin (IL)- 6 and transforming growth factor (TGF)- $\beta 1$ work together to direct $\mathrm{T}$ cells to a Th17 phenotype, whereas 
TGF- $\beta 1$ alone instructs $T$ cells to function as Tregs $(14,15)$. In certain conditions, Tregs may be converted to Th17 cells. Therefore, investigating the gene regulatory mechanisms of Th17 and Treg cell differentiation may contribute to the discovery of crucial factors that are involved in activation of the immune responses in anti-GBM GN.

Inhibitor of DNA binding 3 (ID3) is a $13 \mathrm{kDa}$ nuclear protein that is upregulated in a number of cell types upon stimulation by several growth and differentiation signals $(16,17)$, including $\mathrm{T}$ cell receptor (TCR)- and B cell receptor (BCR)-mediated signals in $\mathrm{T}$ and $\mathrm{B}$ lymphocytes, respectively $(18,19)$. As a member of the basic-helix-loop-helix (bHLH) transcription factor family, ID3 is unique in that it lacks the basic region required for DNA binding (20); however, ID3 retains the functional dimerization domain, and is an inhibitor of the bHLH protein E2A by binding to its target genes and forming inactive heterodimers $(20,21)$. Previous studies reported that ID3, as a transcription factor that is involved in $\mathrm{T}$ cell development, regulates the TGF- $\beta 1$-mediated reciprocal differentiation of Tregs and Th17 cells in mice $(21,22)$. However, how ID3 is involved in the progression of anti-GBM GN and the mechanisms by which ID3 may alter the profiles of Tregs and Th17 cells in anti-GBM GN remain unknown.

The present study demonstrated that the Th17- and Treg cell-mediated immune response contributed to anti-GBM GN in mice. With the development of anti-GBM GN, ID3 involvement in $\mathrm{CD}^{+} \mathrm{T}$ cell differentiation led to a downregulation in Th17 cells, which may affect the delicate balance between Th17 cells and Tregs by skewing CD4 ${ }^{+} \mathrm{T}$ cell differentiation towards Tregs. This effect of ID3 may depend on binding with E2A. The present results suggested that ID3 may protect mice against anti-GBM GN.

\section{Materials and methods}

Mice. Male C57BL/6 mice (age, 6-8 weeks; weight, 20-22 g; $\mathrm{n}=40$ ) were obtained from Beijing HFK Bioscience Co., Ltd. (Beijing, China) and raised in the Animal Care Unit of Tongji Medical College, Huazhong University of Science and Technology (Wuhan, China). All mice were provided with water and a standard laboratory diet ad libitum, and were housed at room temperature $\left(23-26^{\circ} \mathrm{C}\right)$ with $50 \%$ humidity and a 12-h light/dark cycle, receiving humane care in accordance with governmental and institutional guidelines. All experimental procedures were approved by the Animal Care and Use Committee of Tongji Medical College. Each experiment was replicated three times.

Induction of anti-GBM GN in mice. Anti-GBM serum was harvested from rabbits immunized by C57BL/6 mice GBMs, according to standard laboratory protocols (4). All mice were randomly divided into two groups ( $n=20 /$ group), the Control group and the anti-GBM GN group. All mice were preimmunized with a subcutaneous injection of rabbit immunoglobulin (Ig)G (0.02 mg/g; Sigma-Aldrich; Merck KGaA, Darmstadt, Germany) and complete Freund's adjuvant (Sigma-Aldrich; Merck KGaA) 10 days prior to the induction of nephritis. A total of 10 days later, anti-GBM serum $(0.02 \mathrm{ml} / \mathrm{g})$ was administered through the tail vein to induce nephritis in mice in the anti-GBM GN group, whereas the Control group mice received normal rabbit $\mathrm{IgG}(0.02 \mathrm{ml} / \mathrm{g})$. Mice were sacrificed at day $7,14,21$ and 28 .

Renal function analysis. Following sacrifice, blood and urine samples were collected at day 7, 14, 21 and 28, and tested with the Serum Creatinine (SCR) Assay kit and the Urea Assay kit (BioAssay Systems, Hayward, CA, USA). Urine albumin excretion was determined with the Bicinchoninic Acid (BCA) Protein Assay kit (Beyotime Institute of Biotechnology, Shanghai, China). The absorbance of the final reactant was determined at $450 \mathrm{~nm}$ with an ELISA plate reader (BioTek Instruments, Inc., Winooski, VT, USA). All assays were performed according to the kit manufacturer's protocols.

Histology. Following sacrifice, the renal cortex was immediately harvested from each mouse, and fixed with $4 \%$ paraformaldehyde overnight at room temperature, dehydrated in a series of ethanol (80, 95 and $100 \%$, respectively; $5 \mathrm{~min}$ each), and embedded in paraffin. Renal tissues were sectioned $(3 \mu \mathrm{m})$ and stained with periodic acid-Schiff (PAS) reagent at room temperature for $10 \mathrm{~min}$ for histological analysis. Glomerular crescent formation and glomerular sclerosis (deposition of PAS-positive material) were assessed in 30 glomeruli per slide in a blinded manner in PAS-stained paraffin sections. Specimens were examined with an Olympus electron microscope (Olympus Corporation, Tokyo, Japan).

To analyze the deposition of rabbit IgG and mouse IgG in kidneys, snap-frozen renal cortex sections ( $5 \mu \mathrm{m}$; frozen in liquid nitrogen and stored at $-80^{\circ} \mathrm{C}$ ) were fixed in ice cold acetone for $5 \mathrm{~min}$ at $-20^{\circ} \mathrm{C}$, air dried for $30 \mathrm{~min}$, and then were stained directly with fluorescein isothiocyanate (FITC)-conjugated sheep anti-rabbit IgG (cat. no. F6005; 1:100; Sigma-Aldrich; Merck KGaA) and FITC-conjugated sheep anti-mouse IgG (cat. no. F3008; 1:100; Sigma-Aldrich; Merck KGaA) antibodies at room temperature for $25 \mathrm{~min}$. At least 10 cortical interstitial fields (excluding perivascular areas; magnification, $x 400$ ) were examined per sample. Specimens were examined with an Olympus electron microscope (Olympus Corporation).

Leukomonocyte isolation from spleen. Leukomonocytes were isolated from mouse spleens according to a previously published protocol (4). Briefly, tissues from 3 mice per in vitro experiment, were homogenized and sequentially passed through 70 and $40 \mu \mathrm{m}$ nylon mesh. In case of spleen single-cell suspension, erythrocytes were lysed using Red Blood Cell Lysis Buffer (Biolegend, Inc., San Diego, CA, USA). Subsequently, cells were washed several times with PBS and resuspended in RPMI-1640 medium (Gibco; Thermo Fisher Scientific, Inc., Waltham, MA, USA) with $10 \%$ fetal bovine serum (FBS; Thermo Fisher Scientific, Inc.). The viability of the cells was assessed by trypan blue staining prior to fluorescence-activated cell sorting (FACS; as described in the following Flow cytometry subsection).

$C D 4^{+} T$ cell isolation and Treg and Th17 cell differentiation in vitro. $\mathrm{CD}^{+} \mathrm{T}$ cells were isolated from spleens of $\mathrm{C} 57 \mathrm{BL} / 6$ mice by negative magnetic cell sorting (MACS) using the EasySep Mouse CD4 ${ }^{+} \mathrm{T}$ Cell Enrichment kit (Stemcell Technologies, Inc., Vancouver, BC, Canada), according to 
manufacturer's protocol. The purity of $\mathrm{CD} 4^{+} \mathrm{T}$ cells was $>90 \%$, as confirmed by FITC-conjugated anti-CD4 staining and subsequent FACS analysis (as described in the following Flow cytometry subsection). Purified $\mathrm{CD}^{+} \mathrm{T}$ cells were incubated with $10 \mu \mathrm{M}$ carboxy-fluorescein diacetate, succinimidyl ester (CFDA SE) from the Vybrant CFDA SE Cell Tracer kit (Invitrogen; Thermo Fisher Scientific, Inc.), according to manufacturer's protocol, and subsequently cultured in 96-well plates with RPMI-1640 (Gibco; Thermo Fisher Scientific, Inc.) supplemented with $10 \%$ FBS (Thermo Fisher Scientific, Inc.), anti-CD3 $(2.5 \mu \mathrm{g} / \mathrm{ml})$ and anti-CD28 $(5 \mu \mathrm{g} / \mathrm{ml})$ at $37^{\circ} \mathrm{C}$ in a humidified atmosphere $\left(5 \% \mathrm{CO}_{2}\right) . \mathrm{CD}^{+} \mathrm{T}$ cell proliferation was examined. Purified $\mathrm{CD}^{+}{ }^{+} \mathrm{T}$ cells $\left(2 \times 10^{6}\right.$ cells $/ \mathrm{ml}$; in RPMI-1640 medium with 10\% FBS) were differentiated into Th17 cells or Tregs in the presence of different cytokines and antibodies for 6 days at $37^{\circ} \mathrm{C}$. For Th17 cells differentiation, the culture medium was supplemented with IL-6 $(30 \mathrm{ng} / \mathrm{ml})$, TGF- $\beta 1$ (3 ng/ml), IL-1 $\beta$ (20 ng/ml), tumor necrosis factor- $\alpha$ (20 ng/ml), IL-23 (20 ng/ml), anti-interferon (IFN)- $\gamma(10 \mu \mathrm{g} / \mathrm{ml})$ and anti-IL-4 $(10 \mu \mathrm{g} / \mathrm{ml})$ neutralizing antibodies. For Treg cell differentiation, the cultures were supplemented with TGF- $\beta 1$ (10 ng/ml), anti-IFN- $\gamma(10 \mu \mathrm{g} / \mathrm{ml})$ and anti-IL-4 $(10 \mu \mathrm{g} / \mathrm{ml})$.

$\mathrm{CD}^{+} \mathrm{T}$ cell transfection. Isolated $\mathrm{CD}^{+} \mathrm{T}$ cells $\left(1 \times 10^{6}\right.$ cells/well) were seeded in 12 -well plates and were transfected with $100 \mathrm{nM}$ of either ID3-targeted small interfering (si)RNA (cat. no. sc-38003; Santa Cruz Biotechnology, Inc., Dallas, TX, USA) or Scrambled siRNA (cat. no. sc-37007; Santa Cruz Biotechnology, Inc.; sequences commercially unavailable) using the Amaxa Mouse T Cell Nucleofector kit (cat. no. VPA-1006; Lonza Group, Ltd., Basel, Switzerland), according to the manufacturer's protocol. Cells were gently transferred into 12-well plates (final volume, $2 \mathrm{ml}$ media/well) and incubated for $24 \mathrm{~h}$ at $37^{\circ} \mathrm{C}$; transfected $\mathrm{CD} 4^{+} \mathrm{T}$ cells were harvested by centrifugation at $71.55 \mathrm{x} \mathrm{g}$ for $3 \mathrm{~min}$ at room temperature and the supernatants were discarded. The cells were then washed with PBS and collected for subsequent experiments.

Flow cytometry. For FACS flow cytometric analysis, the following antibodies were used: FITC-conjugated anti-CD4 (cat. no. 11-0041; 1:200); phycoerythrin (PE)-conjugated anti-CD25 (cat. no. 12-0251; 1:100); PE-conjugated anti-IL-17A (cat. no. 12-7177; 1:100) and allophycocyanin-conjugated anti-forkhead box P3 (FoxP3; cat. no. 71-5775; all eBioscience; Thermo Fisher Scientific, Inc.) according to the manufacturer's instructions. All procedures were performed at $4^{\circ} \mathrm{C}$ for $25 \mathrm{~min}$, away from light. For staining of intracellular IL-17A, splenocytes $\left(1 \times 10^{5}\right)$ were incubated at $37^{\circ} \mathrm{C}$ in $5 \% \mathrm{CO}_{2}$ for $5 \mathrm{~h}$ with phorbol 12-myristate 13-acetate $(50 \mathrm{ng} / \mathrm{ml}$; Sigma-Aldrich; Merck KGaA) and ionomycin (1 $\mu \mathrm{g} / \mathrm{ml}$; Calbiochem; Merck $\mathrm{KGaA})$ in RPMI-1640 with $10 \%$ FBS. Brefeldin A $(10 \mu \mathrm{g} / \mathrm{ml}$; Sigma-Aldrich; Merck KGaA) was then added for 30 min at $4^{\circ} \mathrm{C}$. Cells were washed several times over $30 \mathrm{~min}$ with PBS and stained with the cell surface marker FITC-anti-CD4 for $25 \mathrm{~min}$ at $4^{\circ} \mathrm{C}$. Cells were incubated for $20 \mathrm{~min}$ in the dark at room temperature in Fixation Buffer (Biolegend, Inc., San Diego, CA, USA) to fix cell surface marker, and Permeabilization Wash Buffer (Biolegend, Inc.) was used to permeabilize cell membranes. Subsequently, intracellular IL-17A was stained using an PE-anti-mouse IL-17A antibody for $25 \mathrm{~min}$ at $4^{\circ} \mathrm{C}$; endonuclear FoxP3 staining was performed using the anti-mouse FoxP3 Staining kit (eBioscience; Thermo Fisher Scientific, Inc.) according to the manufacturer's protocol. Flow cytometry was performed on a BD FACSCalibur Flow Cytometry System (BD Biosciences, Franklin Lakes, NJ, USA) with FCS Expression v3 software (De Novo Software, Glendale, CA, USA).

ELISA. The levels of IL-17A, IL-10 and TGF- $\beta 1$ in serum and cell supernatants were quantified using ELISA Assay kits (cat. nos. DKW12-2170, DKW12-2100 and DKW12-2710; Dakewe Bioengineering Co., Ltd. Beijing, China), according to the manufacturer's protocol. The absorbance of the final reactant was detected at $450 \mathrm{~nm}$ with an ELISA Microplate Reader (Thermo Fisher Scientific, Inc.).

Western blotting. Nuclear and total protein extracts were obtained from cultured $\mathrm{CD}^{+} \mathrm{T}$ cells (in vitro), kidneys and spleens (in vivo) using Nuclear and Total Protein Extraction kits (Beyotime Institute of Biotechnology), according to the manufacturer's protocol. Protein was determined using a BCA Protein Assay kit (Beyotime Institute of Biotechnology). Equal amounts of cell culture or tissue protein extracts (30 and $60 \mu \mathrm{g}$, respectively) were separated by $10 \%$ SDS-PAGE and blotted onto polyvinylidene fluoride membranes (Thermo Fisher Scientific, Inc.). Following blocking for $1 \mathrm{~h}$ at room temperature with $5 \%$ fat-free dry milk in TBS containing $0.1 \%$ Tween-20, membranes were incubated at $4^{\circ} \mathrm{C}$ overnight with the following primary antibodies: Mouse anti-ID3 (1:250; cat. no. 556524; BD Biosciences); rabbit anti-E2A (1:250; cat. no. AP13991b; Abgent, Inc., San Diego, CA, USA); mouse anti-FoxP3 (1:500; cat. no. AO1042a; Abgent, Inc.); mouse anti-RAR-related orphan receptor (ROR)- $\gamma \mathrm{t}$ (1:250; cat. no. 562663; BD Biosciences); rabbit anti-Lamin A/C (1:2,000; cat. no. ab108922; Epitomics; Abcam, Cambridge, MA, USA) and mouse anti-GAPDH (1:5,000; cat. no. A01020; Wuhan, China). Membranes were then incubated with the following secondary antibodies for $1 \mathrm{~h}$ at $37^{\circ} \mathrm{C}$ : Alkaline phosphatase-conjugated anti-rabbit IgG (cat. no. A120-201AP), anti-rat IgG (cat. no. A110-106AP) or anti-mouse IgG (cat. no. A90-105AP; all Dakewe Bioengineering Co., Ltd.). Specific bands were visualized using a 5-bromo-4-chloro-3'indolyphosphate/nitro-blue tetrazolium Alkaline Phosphatase Color Development kit (Dakewe Bioengineering Co., Ltd.), according to the manufacturer's instructions, under protection from direct light. The optical density of the bands was quantified using Image $\mathbf{J}$ v2.1.4.7 (National Institutes of Health, Bethesda, MD, USA). Protein expression was normalized to that of GAPDH.

Co-immunoprecipitation (IP). Co-IP was performed as previously described (23). Total proteins $(800 \mu \mathrm{g})$ of renal tissues and spleens were homogenized in IP buffer (Tris, $\mathrm{pH} 7.4,10 \%$ glycerol, $1 \%$ Nonidet P-40, protease inhibitors and $500 \mu \mathrm{mol} / 1$ sodium vanadate) containing complete protease inhibitor cocktail and phosphate inhibitor cocktail A (cat. no. sc-45044; Santa Cruz Biotechnology, Inc.), and incubated on ice for $30 \mathrm{~min}$. Supernatants were collected followed by centrifugation $\left(12,000 \mathrm{x}\right.$ g for $5 \mathrm{~min}$ at $\left.4^{\circ} \mathrm{C}\right)$. Total proteins $(800 \mu \mathrm{g})$ was incubated with $4 \mu \mathrm{g}$ of either anti-E2A antibody (cat. no. sc-349; Santa Cruz Biotechnology, Inc.) or rabbit 
IgG (Abmart, Inc., Shanghai, China) on ice for $1 \mathrm{~h}$. Protein A/G PLUS-Agarose Beads (100 $\mu \mathrm{l}$; Abmart, Inc.) were added and the beads bound to the antigen-antibody complex were precipitated overnight in a clinical rotor at $4^{\circ} \mathrm{C}$. The beads were washed with IP buffer extensively, and $0.25 \mathrm{ml}$ of SDS sample loading buffer containing $10 \% \beta$-mercaptoethanol was added to the agarose beads and heated at $100^{\circ} \mathrm{C}$ for $10 \mathrm{~min}$. The denatured proteins were analyzed by western blotting with mouse anti-ID3 (1:250; cat. no. 556524; BD Biosciences).

Revere transcription-quantitative polymerase chain reaction $(R T-q P C R)$. Total RNA was extracted from the splenic and renal tissues of each mouse ( $n=6 /$ group) with TRIzol (Invitrogen; Thermo Fisher Scientific, Inc.). A total of $1 \mu \mathrm{g}$ RNA was used to synthesize cDNA with the GoScript Reverse Transcription System (Promega Corporation, Madison, WI, USA), according to the manufacturer's protocol. The temperature protocol for RT-PCR was as follows: $94^{\circ} \mathrm{C}$ for $2 \mathrm{~min}$, followed by 35 cycles of $94^{\circ} \mathrm{C}$ for $30 \mathrm{sec}, 60^{\circ} \mathrm{C}$ for $30 \mathrm{sec}$ and $72^{\circ} \mathrm{C}$ for $1 \mathrm{~min}$, and then $72^{\circ} \mathrm{C}$ for $5 \mathrm{~min}$. qPCR was performed with $1 \mu \mathrm{l}$ cDNA in the presence of $1.5 \mu \mathrm{l}(0.3 \mu \mathrm{M})$ specific murine primers and $10 \mu \mathrm{l}$ Maxima SYBR-Green qPCR Master Mix (Thermo Fisher Scientific, Inc.). The levels of GAPDH mRNA were measured as an internal standard for calibration. The thermocycling conditions for qPCR were as follows: $95^{\circ} \mathrm{C}$ for $10 \mathrm{~min}$, followed by 40 cycles of $15 \mathrm{sec}$ at $95^{\circ} \mathrm{C}, 15 \mathrm{sec}$ at $58^{\circ} \mathrm{C}$ and $45 \mathrm{sec}$ at $72^{\circ} \mathrm{C}$. Melting curve analysis was also included ( 1 cycle of $95^{\circ} \mathrm{C}$ for $1 \mathrm{~min}, 55^{\circ} \mathrm{C}$ for $30 \mathrm{sec}$ and $95^{\circ} \mathrm{C}$ for $30 \mathrm{sec}$ ), in order to verify the specificity of the amplified PCR products. Primers used were as follows: ID3 forward, 5'-TTAACCCAG CCCTCTTCACTTAC-3' and reverse, 5'-CCATTCTCGGAA AAGCCAGT-3'; E2A forward, 5'-GGATCTGAGGTTAAT GGCTCGCTC-3' and reverse 5'-CCTGCATCGTAGTTG GGGGATAAG-3'; FoxP3 forward, 5'-GCAACTCAAGAT GCTGTCCA-3' and reverse, 5'-GGCTGGAAGAGACAG ACAGG-3'; GAPDH forward, 5'-CATCTCCGCCCCTTC TGC-3' and reverse, 5'-CATCACGCCACAGCTTTCC-3'. The primers for ROR $\gamma$ t were purchased from GeneCopoeia, Inc. (cat. no. MQP030076; Rockville, MD, USA). Quantification was performed using the $2^{-\Delta \Delta C q}$ method (24) and the results were normalized to those of GAPDH.

Statistical analysis. Results are expressed as the mean \pm standard error of the mean. All data were analyzed by Student's t-test ore one-way analysis of variance followed multiple comparisons with Tukey's post hoc test. A value of $\mathrm{P}<0.05$ was considered statistically significant, and all experiments were repeated at least 3 times.

\section{Results}

Th17 and Tregs cell-mediated immune response contributes to anti-GBM GN in mice. To investigate the kinetic profiles of Th17 and Treg cells, anti-GBM GN and Control mice were sacrificed at days 7, 14, 21, 28 following the first immunization, and splenocytes were and analyzed by FACS. Th17 cells $\left(\mathrm{CD} 4{ }^{+} \mathrm{IL}-17 \mathrm{~A}^{+}\right)$and Tregs $\left(\mathrm{CD} 4{ }^{+} \mathrm{CD} 25^{+} \mathrm{FoxP} 3^{+}\right)$were increased in anti-GBM GN mice compared with Control mice (Fig. 1A). Notably, the Th17 cell population significantly increased from day 7 and reached a peak at day 14, whereas Tregs increased significantly from day 14 up to day 28. The infiltration of leukocytes has been associated with the increased expression of certain cytokines in peripheral blood (4). Therefore, quantification of cytokine expression levels in blood samples were evaluated, which revealed high levels of IL-17A, IL-10 and TGF- $\beta 1$ in anti-GBM GN compared with Control (Fig. 1B). In addition, expression levels of the transcription factors FoxP3 and ROR $\gamma \mathrm{t}$ were increased both in renal tissues and spleens (data not shown).

Following the infiltration of Th17 cells and Tregs, the pathology of anti-GBM GN progressed. Renal structural damage analyses in nephritic mice revealed severe glomerular crescent formation from day 21 . In addition, thickening and breakage of the GBM, glomerular mesangial cell and matrix proliferation, protein casts and inflammatory cell infiltration were observed in nephritic mice from day 14 (Fig. 1C). Renal tissue damage worsened with disease progression owing to Th17 and Treg cell-mediated immune responses. Anti-GBM GN treatment induced the deterioration of renal function and led to the deposition of the mouse $\operatorname{IgG}$ in glomeruli at day 7 post-treatment (Fig. 1D and E).

ID3 involves in the differentiation of Th17 cells and Tregs. Purified $\mathrm{CD}^{+} \mathrm{T}$ cells were isolated from the spleens of C57BL/6 mice and stimulated by anti-CD3 and anti-CD28 antibodies with the presence of TGF- $\beta 1$ (differentiation towards Tregs) or TGF- $\beta 1$ plus IL-6 (differentiation towards Th17 cells; Fig. 2A and B). Following 6 days of incubation, the supernatants were collected for ELISA. The expression of IL-10 increased in the presence of TGF- $\beta 1$, and the expression of IL-17A increased with TGF- $\beta 1$ plus IL-6 (Fig. $2 \mathrm{C}$ and D). Cells from the different groups were collected for western blotting and RT-qPCR. The levels of ID3 increased when $\mathrm{CD}^{+} \mathrm{T}$ cells differentiated to Tregs, and were reduced in Th17 cells (Fig. 2E-H). Above all, ID3 may be involved in the differentiation of Th17 cells and Tregs in vitro.

ID3 negatively regulates Th17 cell differentiation. As demonstrated, ID3 may be involved in the differentiation of Th17 and Treg cells; however, it is still unknown how ID3 alters the profiles of these cells. Therefore, ID3 was knocked down using siRNA to investigate the effects of reduced ID3 on CD4 ${ }^{+}$ $\mathrm{T}$ cell differentiation (Fig. 3A and B). Following transfection with ID3-siRNA, the expression level of RoR $\gamma t$ mRNA was significantly increased and the mRNA expression of FoxP3 was decreased compared with expressions in Scram-siRNA transfected cells (Fig. 3C). In addition, a significant increase in the protein expression level of IL-17A was observed when ID3 was knocked down (Fig. 3D), whereas there no significant difference was identified in the protein expression levels of TGF- $\beta 1$ and IL-10 in ID3-siRNA group compared with the Scramble-siRNA control group (Fig. 3D). These data indicated that ID3 may affect the delicate balance between Th17 and Treg cells by skewing the differentiation of $\mathrm{CD}^{+} \mathrm{T}$ cells towards Tregs.

ID3 contributes to the regulation of differentiation, which depends on the binding with E2A. To investigate how ID3 regulates the differentiation of $\mathrm{CD}^{+} \mathrm{T}$ cells, the expression levels of ID3 and E2A in both kidneys and spleens were detected by 
A

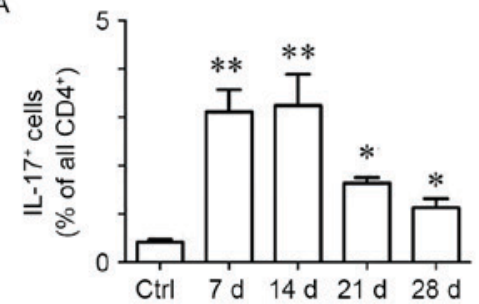

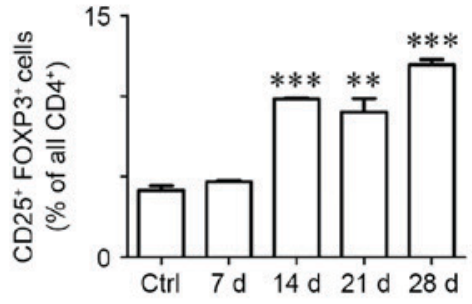

B
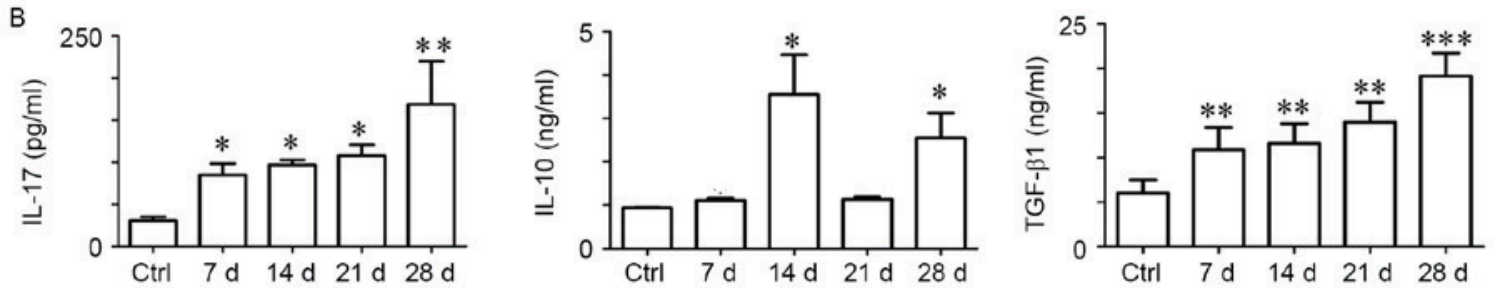

C

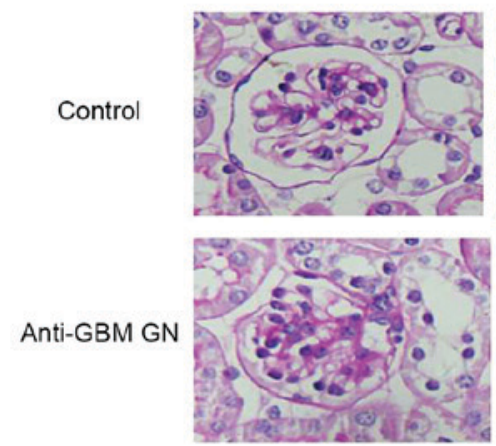

$7 \mathrm{~d}$

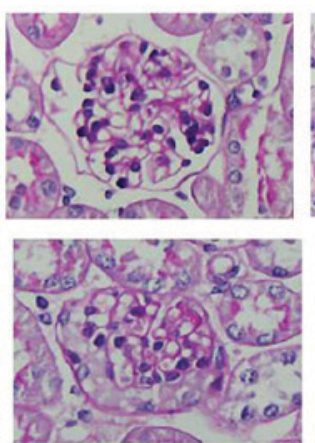

$14 \mathrm{~d}$

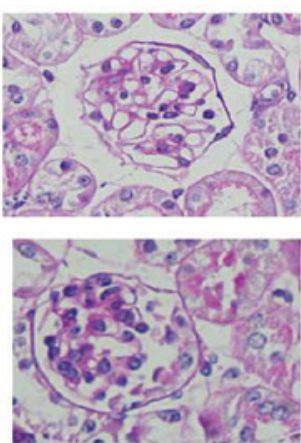

$21 d$
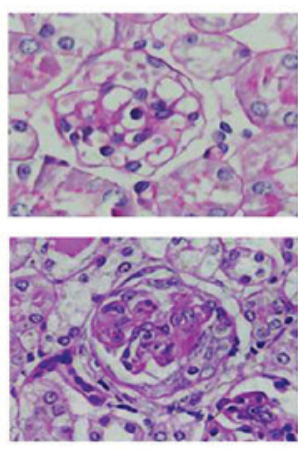

$28 \mathrm{~d}$
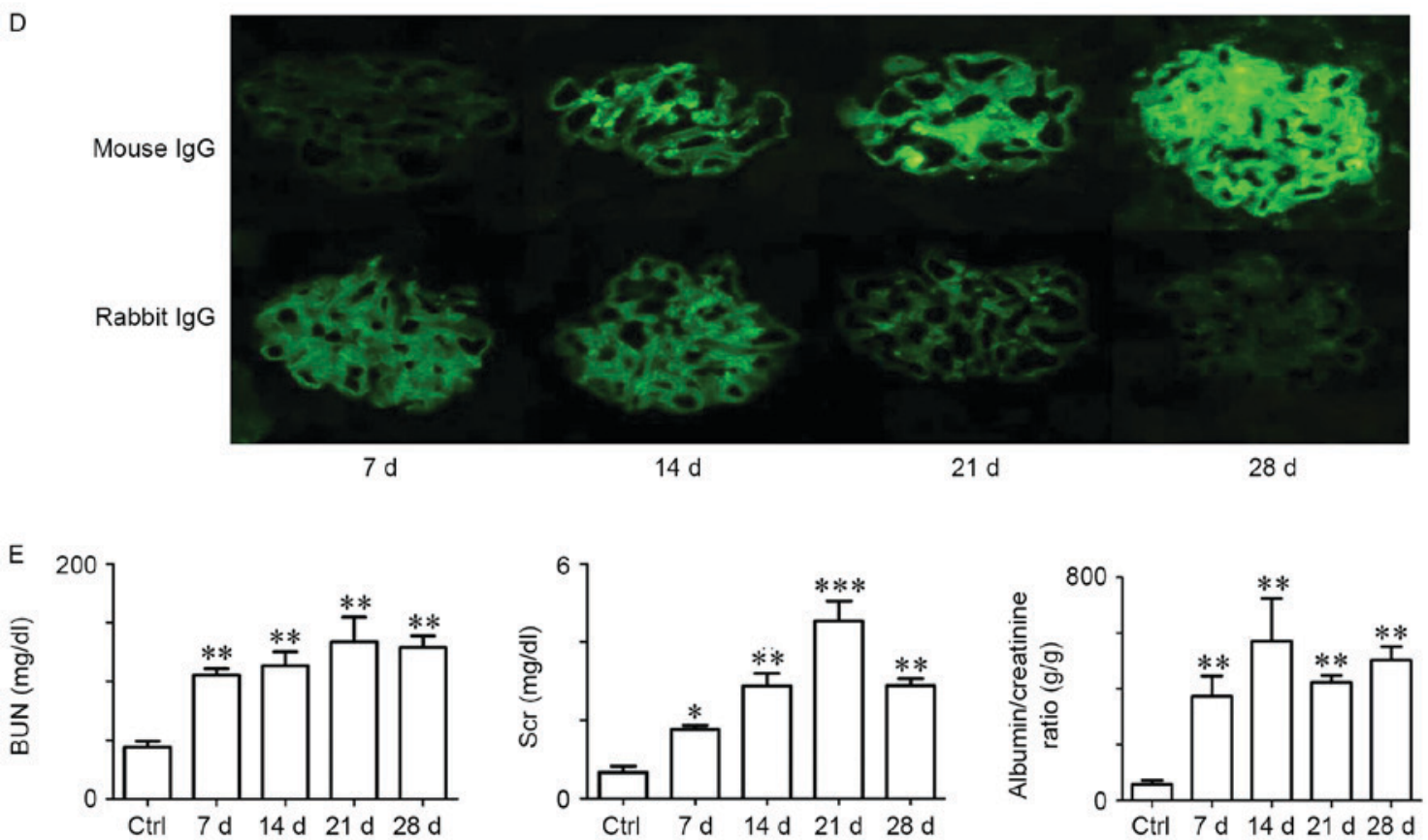

Figure 1. Th17 and Treg cell-mediated immune response contributes to anti-GBM GN in mice. (A) Quantification of the percentage of IL-17A ${ }^{+}$and $\mathrm{CD}^{+} 5^{+}$ FoxP $3^{+}$cells in the $\mathrm{CD}^{+} \mathrm{T}$ cell subset during the time course of the GN. Flow cytometric analysis of splenocytes isolated from Control and anti-GBM GN mice at day 7, 14, 21 and 28 following GN induction. (B) Protein expression levels of IL-17A, IL-10 and TGF- $\beta 1$ in peripheral blood were detected by ELISA in Control and nephritic mice at days 7, 14, 21 and 28. (C) Representative photographs of periodic acid-Schiff-stained kidney sections in Control and nephritic mice at day 7, 14, 21 and 28; magnification, x400. (D) Representative photographs of the deposition of mouse IgG and rabbit IgG in the kidneys. (E) BUN and Scr concentrations and albumin/creatinine ratio in urine from Control and nephritic mice were analyzed in Control and nephritic mice at day $7,14,21$ and 28. Data are presented as the mean \pm standard error of the mean; $\mathrm{n}=3-4 /$ group; ${ }^{*} \mathrm{P}<0.05,{ }^{* *} \mathrm{P}<0.01$ and ${ }^{* * *} \mathrm{P}<0.005$ vs. Control. BUN, blood urea nitrogen; Ctrl, control; d, day; FoxP3, forkhead box P3; GBM, glomerular basement membrane; GN, glomerulonephritis; IgG, immunoglobulin G; IL, interleukin; Scr, serum creatinine; TGF, transforming growth factor; Th17, T helper 17; Treg, regulatory $\mathrm{T}$. 
A

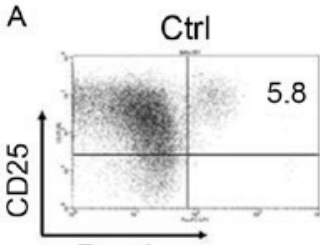

Foxp3

C

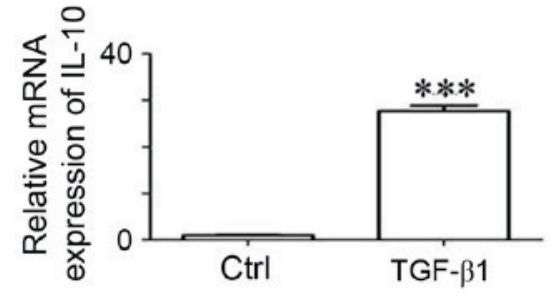

$\mathrm{E}$

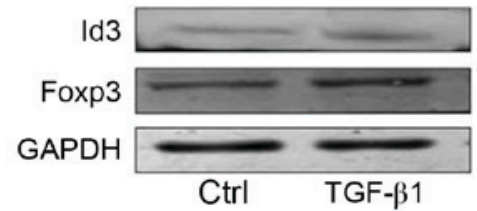

G

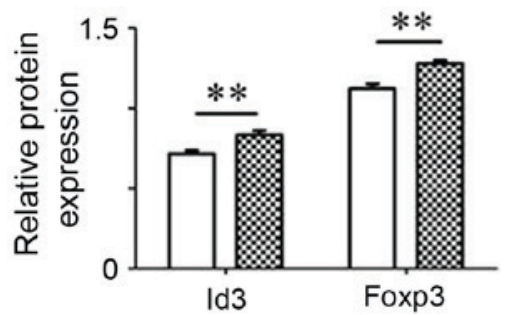

B

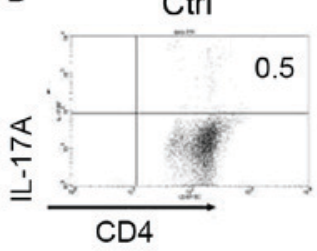

TGF- $\beta 1+I L-6$

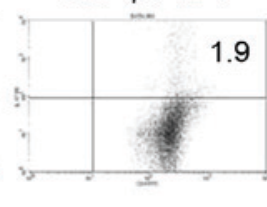

D
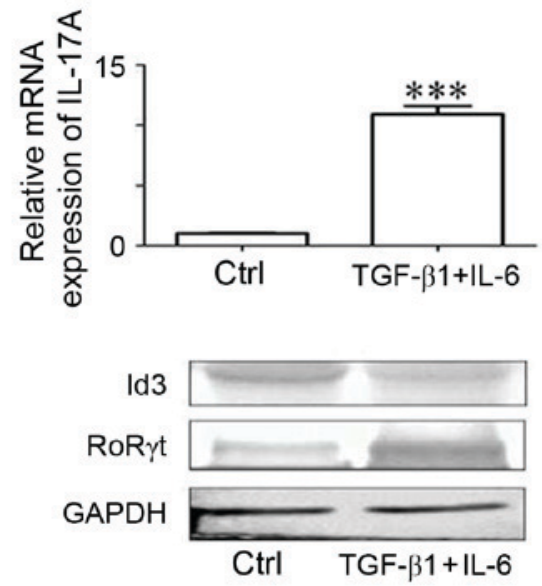

$\mathrm{H}$

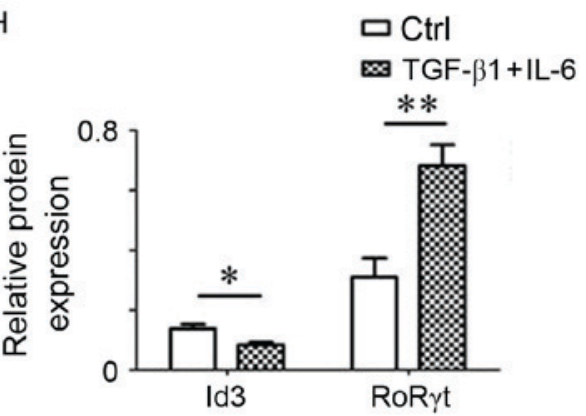

Figure 2. ID3 serves a role the differentiation of Th17 and Treg cells. Purified CD4 ${ }^{+} \mathrm{T}$ cells were isolated from the spleens of C57BL/6 mice and subsequently stimulated with anti-CD3 and anti-CD28 antibodies with or without the presence of TGF- $\beta 1$ or TGF- $\beta 1+$ IL-6. (A and B) Representative gated data plots for $\mathrm{CD} 4^{+} \mathrm{T}$ cells. (C and D) The expression levels of IL-10 and IL-17 were detected in the splenic cell culture supernatants by ELISA. (E and F) Western blotting was used to measure the protein expressions of ID3, FoxP3 and ROR $\gamma \mathrm{t}$ in Th17 and Treg cells. (G and H) Bar graphs indicating the quantification of ID3, FoxP3 and ROR $\gamma \mathrm{t}$ protein expression from $(\mathrm{G}$ and $\mathrm{H})$ Data are present as the mean \pm standard error of the mean of three independent experiments; ${ }^{*} \mathrm{P}<0.05$, ${ }^{* *} \mathrm{P}<0.01$, ${ }_{* * * *} \mathrm{P}<0.005$ vs. Ctrl group. Ctrl, control; FoxP3, forkhead box P3; ID3, inhibitor of DNA binding 3; IL, interleukin; TGF, transforming growth factor; ROR $\gamma \mathrm{t}$, RAR-related orphan receptor $\gamma \mathrm{t}$; Th17, T helper 17; Treg, regulatory T.

RT-qPCR and western blotting. Renal ID3 mRNA expression increased between 3 - and 20-fold from day 7 post-treatment, with a peak at day 14 , compared with the Control group (Fig. 4A). Notably, splenic ID3 mRNA expression decreased significantly in the early phase (day 7 ) and gradually increased in the later phase (day 14 and 21; Fig. 4B). ID3 protein expression was also upregulated in the kidney on day 7 (Fig. 4C), and the protein expression of ID3 was increased in spleen of nephritic mice, (Fig. 4D).

In renal tissues of anti-GBM GN mice, E2A mRNA expression increased in the early phase and then decreased at day 21 (Fig. 4A). However, E2A mRNA expression did not exhibit significant differences in the spleen (Fig. 4B), nor was a significant difference identified in E2A protein expression in kidney or spleen (Fig. 4C and D, respectively). A previous study reported that ID3 regulated the maturation of T cells in the thymus by binding to the transcription factor E2A, forming a non-DNA-binding dimer (25). Therefore, the present study examined the relationship between ID3 and E2A at different phases in anti-GBM GN progression by co-IP. The results indicated that ID3 was able to interact with E2A (Fig. 4E and $\mathrm{F}$ ). In addition, a significant increase in binding between ID3 and E2A in anti-GBM GN was observed from day 7, which declined at the later time points in both renal tissues and spleens.

\section{Discussion}

Anti-GBM glomerulonephritis is a type of autoimmune disease that is frequently associated with systemic and organ-specific autoimmunity (3). There are two phases in anti-GBM GN pathogenesis, the heterologous phase and the autologous phase (26). The heterologous phase corresponds to the effects in the early phase of disease pathology, and the autologous phase gradually dominates with the progression of anti-GBM GN, which was characterized by the immune response of the host against the heterologous antibodies due to the activated $\mathrm{CD} 4^{+} \mathrm{T}$ cells and $\mathrm{B}$ cells (27). As expected, two 


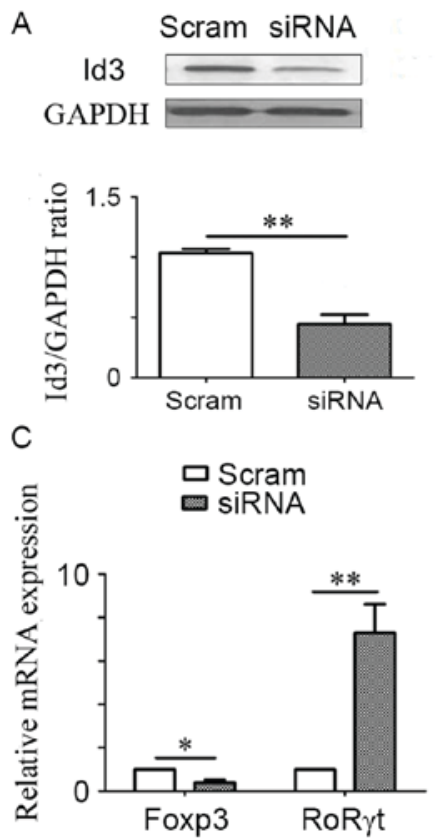

B
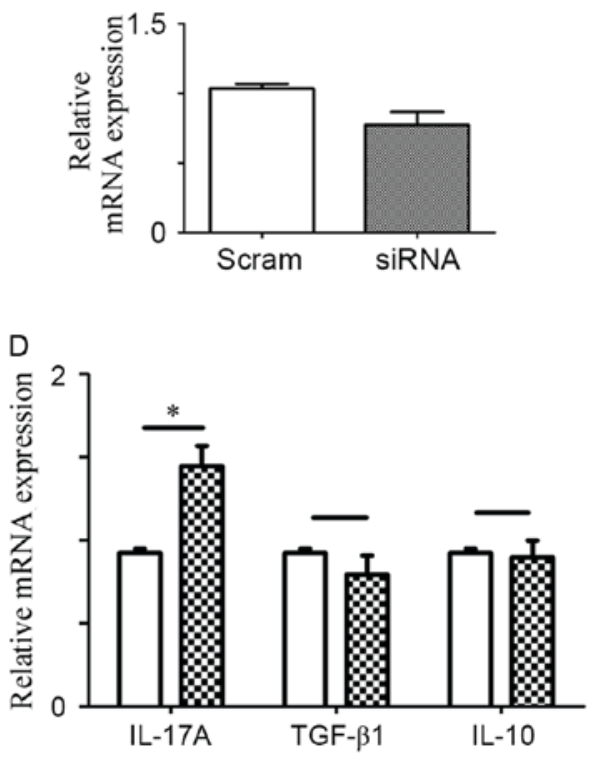

Figure 3. ID3 negatively regulates Th17 cell differentiation. Purified CD4 ${ }^{+} \mathrm{T}$ cells were isolated from the spleens of C57BL/6 mice and treated with ID3-specific siRNA or Scram-siRNA; subsequently, the expression of ID3 (A) protein and (B) mRNA was measured. (C) Following ID3-siRNA or Scram-siRNA treatment, CD4 ${ }^{+} \mathrm{T}$ cells were activated with anti-CD3 and anti-CD28 for $24 \mathrm{~h}$. Cells were harvested to assess the mRNA expression levels of FoxP3 and ROR $\gamma \mathrm{t}$ mRNA. (D) Cell culture supernatants were used to measure the protein expression levels of IL-17A, TGF- $\beta 1$ and IL-10 by ELISA. Data are presented as the mean \pm standard error of the mean of three independent experiments; " $\mathrm{P}<0.05$ and ${ }^{* *} \mathrm{P}<0.01$. FoxP3, forkhead box P3; ID3, inhibitor of DNA binding 3; IL, interleukin; ROR $\gamma t$, RAR-related orphan receptor $\gamma \mathrm{t}$; scram, scrambled; siRNA, small interfering RNA; TGF, transforming growth factor; Th17, T helper 17.

distinct immune phases were observed in anti-GBM GN, and the autologous phase seemed to be more important. $\mathrm{CD}^{+} \mathrm{T}$ cells serve a crucial role in the initiating the immune response, which leads to the crescentic injury in anti-GBM GN $(28,29)$. Our previous study revealed that anti-GBM GN may be driven by Th1 and Th17 cell-mediated immune response (4). The time of Th17 cell-mediated immune response is early in disease pathogenesis and is followed later by Th1 cell-mediated injury (9). Tregs serve a protective role in anti-GBM GN and may significantly reduce renal injury (10). The activity of Th1 and Th17 cells may be attenuated by the anti-inflammatory action of Tregs, which inhibit $\mathrm{T}$ cell proliferation and $\mathrm{T}$ effector function (11). In the present study, the number of Th17 cells appeared to increase from day 7 and reached a peak at day 14 , whereas the number of Tregs increased significantly from day 14 to day 28 . However, although the infiltration of Th17 cells was reduced, the renal tissues continued to deteriorate. These results indicated that activated Th17 cells may be able to initiate and amplify glomerular inflammation, which is in agreement with a previous study (30). The protection of Tregs was not enough to lead to a recovery from autoimmune tissue damage in anti-GBM GN. Thus, it is necessary to identify an effective method that is able to suppress Th17 cells and increase Tregs, in order to exert a regulatory function to protect the kidneys.

Although Th17 and Treg cells have different functions, they have the same precursor cell, which may be differentiated to different cell types by pleiotropic cytokines $(12,13)$. Under certain conditions, Tregs may be converted to Th17 cells; therefore, investigating the gene regulatory mechanisms of Th17 and Treg cell differentiation may contribute to the identification of crucial factors that are involved in activation of the immune responses in anti-GBM GN. A previous study demonstrated that ID3, as a transcription factor that is involved in $\mathrm{T}$ cell development, regulated TGF- $\beta 1$-mediated reciprocal differentiation of Treg and Th17 cells in mice (21). ID3 is a nuclear protein and contains a basic-helix-loop-helix (bHLH) domain, and is upregulated in a number of cell types upon stimulation by several growth and differentiation signals $(16,17)$, including TCR- and BCR-mediated signals in T and B lymphocytes, respectively $(18,19)$. Therefore, the present study hypothesized that ID3 may offer protection in mice against anti-GBM GN by regulating the differentiation of Th17 and Treg cells. The present data indicated that ID3 expression increased when $\mathrm{CD}^{+} \mathrm{T}$ cells differentiated into Tregs, but decreased in Th17 cells in vitro. Th17 and Treg cells were present throughout the entire period of anti-GBM GN, which may differ at different time points accompanied with the expression of ID3. These resulted suggested that ID3 may be involved in the differentiation of Th17 and Treg cells in anti-GBM GN. In addition, the expression levels of RoR $\gamma$ t and IL-17A were increased when ID3 expression was knocked down. No significant difference in the expression of TGF- $\beta 1$ and IL-10 between the ID3-siRNA group and Scramble-siRNA control group. In other words, ID3 may tilt the delicate balance between Th17 and Treg cells by skewing $\mathrm{CD} 4^{+} \mathrm{T}$ cell differentiation towards Tregs, which is of great importance for maintaining tolerance of and the suppressors of anti-GBM GN (10). However, glomerular crescents and protein casts were still observed at day 21 and 28 post-treatment, which led to the deterioration of renal function. This suggested that endogenous ID3 was not enough to reduce renal injury in anti-GBM GN and in vivo administration of exogenous ID3 in mice may be required to increase the number of Tregs in order to protect renal tissues. 
A

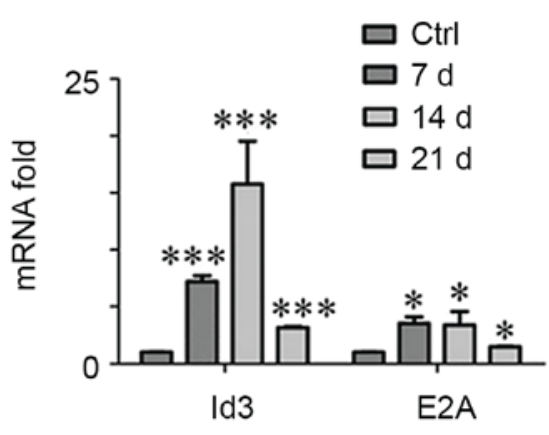

C

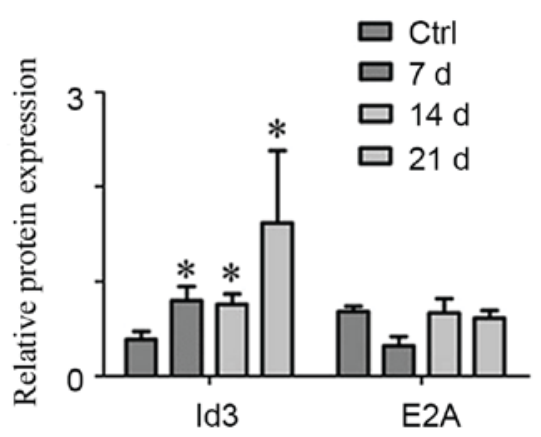

E

IB

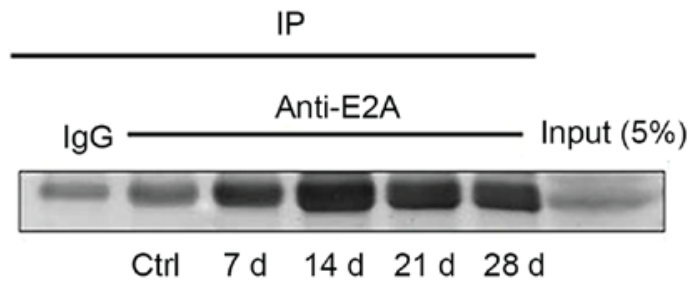

F

IB

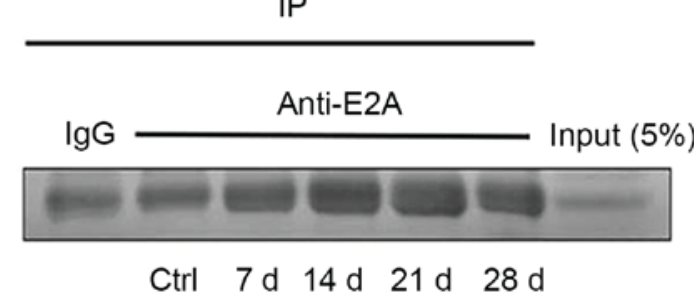

B
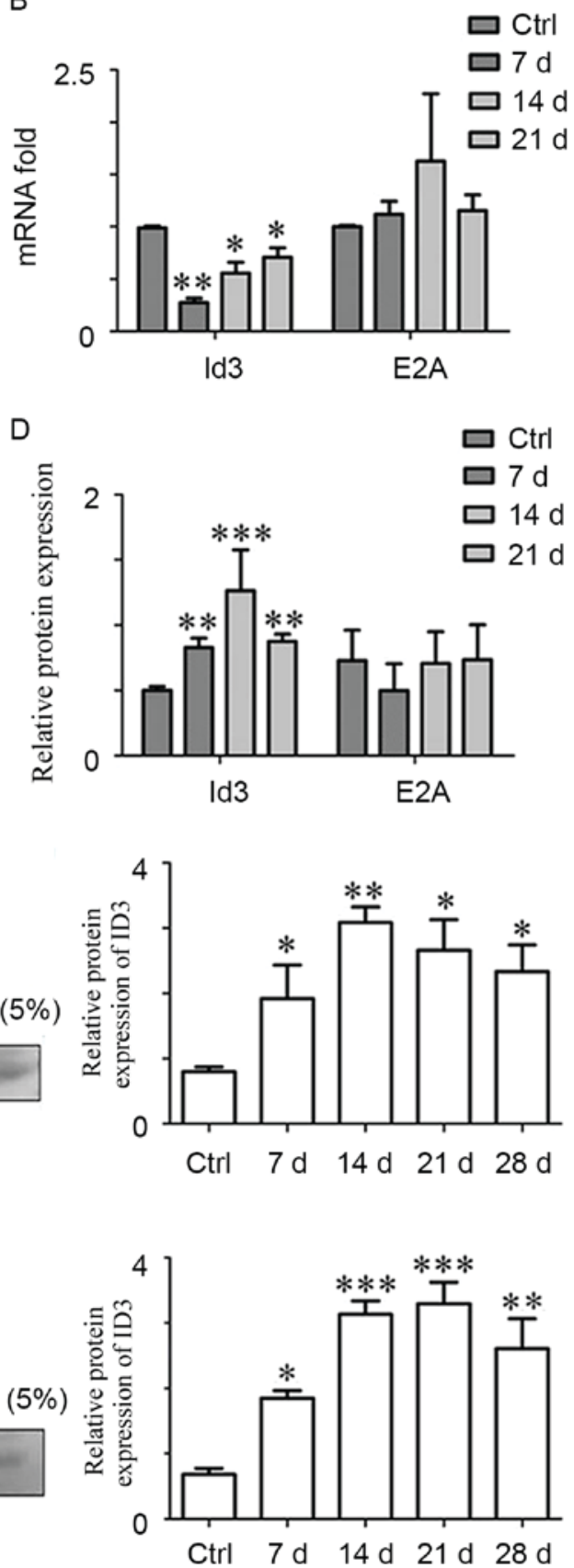

Figure 4. ID3 contributes to the regulation of differentiation, which depends on binding with E2A. (A and B) mRNA expression levels of ID3 and E2A were measured in both (A) kidneys and (B) spleens. (C and D) ID3 and E2A protein expression levels were measured in both (C) kidneys and (D) spleens. Protein extracts from (E) kidneys and (F) spleens were subjected to immunoprecipitation with either anti-E2A antibody or normal rabbit IgG, followed by immunoblotting with anti-ID3 antibodies; the bar graphs represent the quantification of ID3 by Image J. Data are presented as the mean \pm standard error of the mean of at least 3 independent experiments; ${ }^{*} \mathrm{P}<0.05,{ }^{* *} \mathrm{P}<0.01$ and ${ }^{* * * *} \mathrm{P}<0.005$ vs. Control. Ctrl, Control; ID3, inhibitor of DNA binding 3 ; IgG, immunoglobulin G.

To further explore how ID3 affected the differentiation of Th17 and Treg cells, the expression of E2A in kidney and spleen was detected by RT-qPCR and western blotting. A previous study reported that ID3 interacted with the transcription factor E2A to form a non-DNA-binding dimer, which regulates the maturation of T cells in the thymus (27). Consistent with those observations, the present study demonstrated that ID3 binds with E2A in anti-GBM GN. Furthermore, it was observed that the binding between ID3 and E2A increased from day 7 and declined at later period in both renal tissues and spleens. In peripheral lymphoid organs, E2A binds with the promoters to induce the expression of FoxP3 and RoR $\gamma \mathrm{t}$, which are the key transcription factors involved in Treg and Th17 cell differentiation $(21,31,32)$. ID3 may prevent E2A binding to the promoters by forming a non-DNA-binding dimer with E2A (21). Therefore, the present study hypothesized that ID3 may affect the expression of FoxP3 and RoR $\gamma \mathrm{t}$ by binding with E2A, and subsequently regulating the differentiation of Th17 cells and Tregs. However, a limitation to the present study was that ID3 expression was not knocked down in in vivo experiments, which may provide direct evidence to support this hypothesis in our future studies. 
In conclusion, the present study was the first, to the best of our knowledge, to demonstrate the importance of ID3 in anti-GBM GN, and that the Th17 and Treg cell-mediated immune response contributed to anti-GBM GN in mice. As the development of anti-GBM GN progressed, ID3 affected the differentiation of Th17 and Treg cells by downregulating the differentiation of Th17 cells, which may subsequently affect the delicate balance between these cells by skewing $\mathrm{CD}^{+} \mathrm{T}$ cell differentiation towards Tregs; and effect that is probably associated with ID3 binding with E2A.

\section{Acknowledgements}

The present study was supported by The National Science Foundation of China (grant nos. 81241025, 81370817 and 81500546).

\section{References}

1. Pedchenko V, Bondar O, Fogo AB, Vanacore R, Voziyan P, Kitching AR, Wieslander J, Kashtan C, Borza DB, Neilson EG, et al: Molecular architecture of the Goodpasture autoantigen in anti-GBM nephritis. N Engl J Med 363: 343-354, 2010.

2. Phoon RK, Kitching AR, Odobasic D, Jones LK, Semple TJ and Holdsworth SR: T-bet deficiency attenuates renal injury in experimental crescentic glomerulonephritis. J Am Soc Nephrol 19: 477-485, 2008.

3. Tipping PG and Holdsworth SR: T cells in crescentic glomerulonephritis. J Am Soc Nephrol 17: 1253-1263, 2006.

4. Zhang Q, Luan H, Wang L, He F, Zhou H, Xu X, Li X, Xu Q, Niki T, Hirashima M, et al: Galectin-9 ameliorates anti-GBM glomerulonephritis by inhibiting Th1 and Th17 immune responses in mice. Am J Physiol Renal Physiol 306: F822-F832, 2014.

5. Hünemörder S, Treder J, Ahrens S, Schumacher V, Paust HJ, Menter T, Matthys P, Kamradt T, Meyer-Schwesinger C, Panzer U, et al: TH1 and TH17 cells promote crescent formation in experimental autoimmune glomerulonephritis. J Pathol 237: 62-71, 2015.

6. Mosmann TR, Cherwinski H, Bond MW, Giedlin MA and Coffman RL: Two types of murine helper T cell clone. I. Definition according to profiles of lymphokine activities and secreted proteins. J Immunol 136: 2348-2357, 1986

7. Harrington LE, Hatton RD, Mangan PR, Turner H, Murphy TL, Murphy KM and Weaver CT: Interleukin 17-producing CD4+ effector $\mathrm{T}$ cells develop via a lineage distinct from the $\mathrm{T}$ helper type 1 and 2 lineages. Nat Immunol 6: 1123-1132, 2005.

8. Sakaguchi S, Sakaguchi N, Asano M, Itoh M and Toda M: Immunologic self-tolerance maintained by activated $\mathrm{T}$ cells expressing IL-2 receptor alpha-chains (CD25). Breakdown of a single mechanism of self-tolerance causes various autoimmune diseases. J Immunol 155: 1151-1164, 1995.

9. Kitching AR and Holdsworth SR: The emergence of TH17 cells as effectors of renal injury. J Am Soc Nephrol 22: 235-238, 2011

10. Fehérvari Z and Sakaguchi S: CD4+ Tregs and immune control. J Clin Invest 114: 1209-1217, 2004.

11. Barbi J, Pardoll D and Pan F: Metabolic control of the Treg/Th17 axis. Immunol Rev 252: 52-77, 2013.

12. Campbell DJ and Koch MA: Phenotypical and functional specialization of FOXP3+ regulatory T cells. Nat Rev Immunol 11: 119-130, 2011.
13. Yang XO, Nurieva R, Martinez GJ, Kang HS, Chung Y, Pappu BP, Shah B, Chang SH, Schluns KS, Watowich SS, et al: Molecular antagonism and plasticity of regulatory and inflammatory $\mathrm{T}$ cell programs. Immunity 29: 44-56, 2008.

14. Zhu J, Yamane H and Paul WE: Differentiation of effector CD4 T cell populations $\left({ }^{*}\right)$. Annu Rev Immunol 28: 445-489, 2010.

15. Lee YK, Mukasa R, Hatton RD and Weaver CT: Developmental plasticity of Th17 and treg cells. Curr Opin Immunol 21: 274-280, 2009.

16. Norton JD: ID helix-loop-helix proteins in cell growth, differentiation and tumorigenesis. J Cell Sci 113: 3897-3905, 2000.

17. Nakatsukasa H, Zhang D, Maruyama T, Chen H, Cui K, Ishikawa M, Deng L, Zanvit P, Tu E, Jin W, et al: The DNA-binding inhibitor Id3 regulates IL-9 production in CD4(+) T cells. Nat Immunol 16: 1077-1084, 2015.

18. Bain G, Cravatt CB, Loomans C, Alberola-lla J, Hedrick SM and Murre C: Regulation of the helix-loop-helix proteins, E2A and ID3, by the Ras-ERK MAPK cascade. Nat Immunol 2: 165-171, 2001.

19. Pan L, Sato S, Frederick JP, Sun XH and Zhuang Y: Impaired immune responses and B-cell proliferation in mice lacking the ID3 gene. Mol Cell Biol 19: 5969-5980, 1999.

20. Murre C: Helix-loop-helix proteins and lymphocyte development. Nat Immunol 6: 1079-1086, 2005.

21. Maruyama T, Li J, Vaque JP, Konkel JE, Wang W, Zhang B, Zhang $\mathrm{P}$, Zamarron BF, Yu D, Wu Y, et al: Control of the differentiation of regulatory $\mathrm{T}$ cells and $\mathrm{T}(\mathrm{H}) 17$ cells by the DNA-binding inhibitor ID3. Nat Immunol 12: 86-95, 2011.

22. Miyazaki M, Miyazaki K, Chen S, Itoi M, Miller M, Lu LF, Varki N, Chang AN, Broide DH and Murre C: Id2 and ID3 maintain the regulatory $\mathrm{T}$ cell pool to suppress inflammatory disease. Nat Immunol 15: 767-776, 2014.

23. Kee BL, Quong MW and Murre C: E2A proteins: Essential regulators at multiple stages of B-cell development. Immunol Rev 175: 138-149, 2000.

24. Livak KJ and Schmittgen TD: Analysis of relative gene expression data using real-time quantitative PCR and the 2(-Delta Delta C(T)) method. Methods 25: 402-408, 2001.

25. Jones ME and Zhuang Y: Acquisition of a functional $\mathrm{T}$ cell receptor during T lymphocyte development is enforced by HEB and E2A transcription factors. Immunity 27: 860-870, 2007.

26. Le Hir M: Histopathology of humorally mediated anti-glomerular basement membrane (GBM) glomerulonephritis in mice. Nephrol Dial Transplant 19: 1875-1880, 2004.

27. Abbas AK, Murphy KM and Sher A: Functional diversity of helper T lymphocytes. Nature 383: 787-793, 1996.

28. Tipping PG, Huang XR, Qi M, Van GY and Tang WW: Crescentic glomerulonephritis in CD4- and CD8-deficient mice. Requirement for CD4 but not CD8 cells. Am J Pathol 152: 1541-1548, 1998.

29. Huang XR, Tipping PG, Shuo L and Holdsworth SR: Th1 responsiveness to nephritogenic antigens determines susceptibility to crescentic glomerulonephritis in mice. Kidney Int 51: 94-103, 1997.

30. Robertson J, Wu J, Arends J, Zhou C, Adrogue HE, Chan JT and Lou Y: Spontaneous recovery from early glomerular inflammation is associated with resistance to anti-GBM glomerulonephritis: Tolerance and autoimmune tissue injury. J Autoimmun 30: 246-256, 2008.

31. Rivera RR, Johns CP, Quan J, Johnson RS and Murre C: Thymocyte selection is regulated by the helix-loop-helix inhibitor protein, ID3. Immunity 12: 17-26, 2000.

32. Zhang F, Fuss IJ, Yang Z and Strober W: Transcription of ROR $\gamma \mathrm{t}$ in developing Th17 cells is regulated by E-proteins. Mucosal Immunol 7: 521-532, 2014. 